\title{
Diagnostic approach to pulmonary embolism and lessons from a busy acute assessment unit in the UK
}

The diagnosis of pulmonary embolism (PE) can be very elusive and, if missed, may have fatal consequences. Conversely, PE can be over-diagnosed, with the concomitant risks associated with unnecessary anticoagulation. Although there are many tests that used in the diagnosis of $\mathrm{PE}$, no test can exclude this condition with $100 \%$ certainty, and $\mathrm{PE}$ has been reported even after a negative pulmonary angiography. The diagnosis of $\mathrm{PE}$ depends on the interpretation of the available tests in the context of pre-test clinical probabilities. Ventilation/perfusion $\left(V^{\prime} / Q^{\prime}\right)$ scan and computerised tomographic pulmonary angiography (CTPA) are the main screening tests used for patients with suspected PE. However, both $V^{\prime} / Q^{\prime}$ scan and CTPA have to be supplemented by other diagnostic modalities because of their diagnostic limitations. This article reviews the literature concerning the diagnosis of $\mathrm{PE}$, with particular reference to the approach in our acute assessment unit. We conclude by describing two learning points from real cases presenting with suspected $\mathrm{PE}$, in order to highlight how the diagnosis can be missed or made inaccurately.

Despite many diagnostic modalities, the diagnosis of pulmonary embolism (PE) remains very challenging, and PE can be missed with potentially serious consequences [1-3]. Conversely, PE can be over-diagnosed and unnecessary anticoagulation administered, resulting in patients having an erroneous PE label, which could influence management in the event of a similar episode, or if future surgery or pregnancy arises. Unlike myocardial infarction, there is no single test, including pulmonary angiography, which can rule out PE with absolute confidence [4]. As a consequence, the diagnosis of PE should be based on the combination of pretest clinical probability and the results of the special diagnostic tests [5-8].

\section{Clinical probability}

Determination of clinical probability of PE depends on two components: 1) the presence or absence of risk factors [8]; and 2) the presence or absence of an alternative diagnosis which would explain the clinical presentation. There are currently several validated schemes used to determine clinical probability of WeLLS and co-workers [9-11] introduced a validated scoring system based on clinical presentation and the presence or absence of risk factors (table 1). A simpler system was recommended by the British Thoracic Society and validated against other scoring systems (fig. 1) $[8,12]$. According to this system, the absence of a risk factor plus the presence of another condition that could explain the clinical presentation would indicate low clinical probability for PE. However, if there is a risk factor and the clinical presentation cannot be explained by an alternative diagnosis, a high clinical probability for PE should be considered. Intermediate clinical probability for $\mathrm{PE}$ is indicated by the combination of the absence of a risk factor as well as an alternative diagnosis, or the combined presence of a risk factor as well as
M.M.A. Hamad

P. Bhatia ${ }^{2}$

E. Ellidir ${ }^{1}$

M.M. Abdelaziz

V. Connolly ${ }^{1}$

${ }^{1}$ Dept of Acute Medicine, James Cook University Hospital,

Middlesbrough, UK

${ }^{2}$ Dept of Medicine, Tameside

Hospital, Ashton-under-Lyne, UK

\section{Correspondence}

M.M. Abdelaziz

Dept of Medicine

Tameside Hospital

Ashton-under-Lyne, OL6 9RW, UK, muntasirabdelaziz@hotmail.com
HERMES syllabus link: module B.5.1 
Table 1 How to assess clinical probability according to the scoring system of WeLLS et al. [11]

$\begin{array}{lc}\text { Clinical history and signs } & \text { Score } \\ \text { Suspected DVT } & 3.0 \\ \text { An alternative diagnosis is less likely than PE } & 3.0 \\ \text { Heart rate }>\text { 100 beats per min } & 1.5 \\ \text { Immobilisation or surgery in the previous 4 weeks } & 1.5 \\ \text { Previous DVT or PE } & 1.5 \\ \text { Haemoptysis } & 1.0 \\ \text { Malignancy (on treatment, treated in the past } 6 \text { months or palliative) } & 1.0\end{array}$

$\begin{array}{lcc}\text { Risk of having PE } & \text { Score range } & \text { Mean probability of PE \% } \\ \text { Low probability } & <2 & 3.6 \\ \begin{array}{l}\text { Intermediate } \\ \text { probability }\end{array} & 2-6 & 20.5 \\ \text { High probability } & >6 & 66.7 \\ \text { DVT: deep venous thrombosis; PE: pulmonary embolism. } & \end{array}$

an alternative diagnosis $[2,8,12]$. Determining clinical probability does not only improve the diagnostic outcome of the different test used, but can be costeffective, as it assists the decisions for imaging and further investigations.

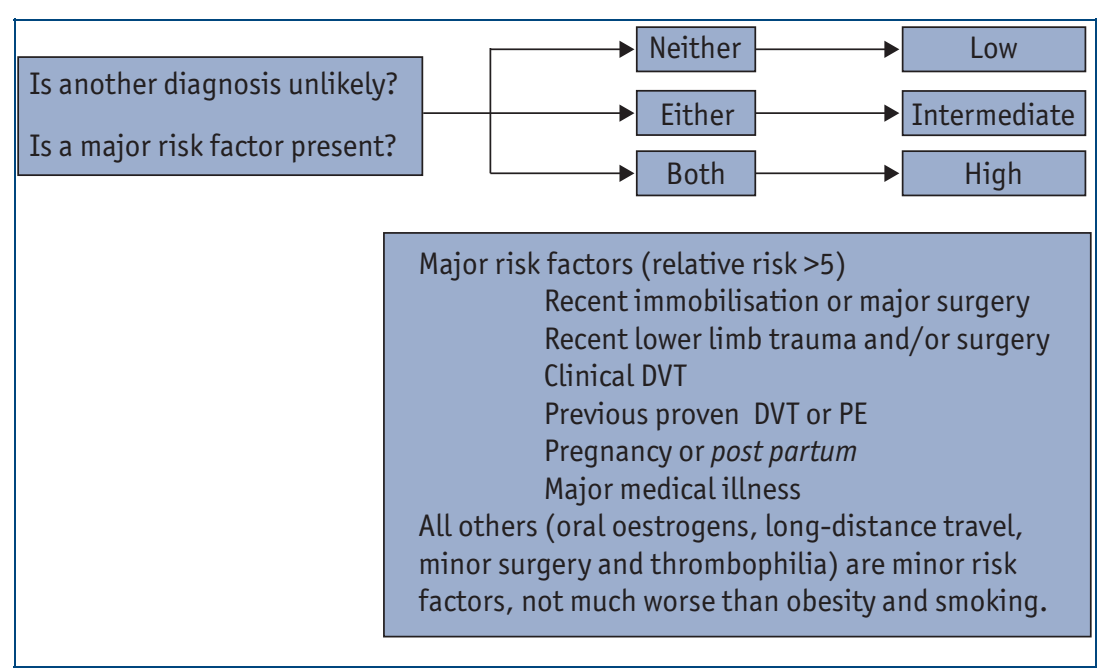

\section{Figure 1}

How to assess clinical probability. Pulmonary embolism (PE) must be a reasonable possibility. Low clinical probability: no risk factor + presence of alternative diagnosis. high clinical probability: risk factor present + no alternative diagnosis; intermediate clinical probability: no risk factor + no alternative diagnosis or risk factor present + presence of alternative diagnosis. DVT: deep venous thrombosis. Modified from the BTS Pulmonary Embolism Handbook, which was developed for British Thoracic Society/Royal College of Physicians Conference, April 7, 2005, "Clinical Problems in Acute Venous Thrombo-Embolism" (A. Miller, Croydon Health Services, Croydon, UK; personal communication).

\section{Diagnostic tests}

There are several investigations used to establish the diagnosis of PE (table 2). The findings of some of these tests, such as ECG, chest radiography (CXR) and arterial blood gases $(A B G s)$, are nonsensitive and nonspecific, and may be misleading (table 2 and fig. 2). Although these tests should be included in the investigations of PE to improve the determination of the clinical probability, the diagnosis of PE should not be made solely on the basis of these investigations. However, diagnostic tests such as D-dimer, compression ultrasound (CUS) of the lower limbs, ventilation/perfusion $\left(V^{\prime} / Q^{\prime}\right)$ scan, computerized tomographic pulmonary angiography (CTPA) and pulmonary angiography, are used after determining the clinical probability to rule in or out the diagnosis of PE. Cardiac biomarkers (troponin-T and -I) and echocardiography can have some prognostic value and may help in assessing the severity of PE.

\section{D-dimer}

D-dimer, a proteolytic derivative of degraded fibrin, can be detected in venous thromboembolism (VTE) as well as many other conditions, including inflammatory disorders, trauma, infections and neoplasia $[13,14]$. Although a positive D-dimer test has no significant diagnostic value, a negative D-dimer test has a high negative predictive value and makes the diagnosis of PE unlikely. The sensitivity of the D-dimer test is significantly improved when combined with the pretest clinical probability. There are different ways of measuring D-dimer, including latex agglutination, red blood cell (RBC) agglutination and ELISA $[13,15,16]$. Latex agglutination is the least sensitive and most specific, while ELISA is the most sensitive and least specific [14, 17-19]. RBC agglutination has an intermediate sensitivity and specificity $[10,14,20,21]$. As the D-dimer test is used primarily to exclude, rather than confirm, the diagnosis of $P E$, an assay with low sensitivity, such as latex agglutination, is not recommended. However, MDA is a new latex agglutination test with a very high sensitivity, comparable to ELISA, and can be used in excluding the diagnosis of PE [22]. Studies have demonstrated that the combination of a negative SimpliRED, ELISA or MDA with a low clinical probability can be used to exclude $\mathrm{PE}$ without the need of further imaging $[8,10,21$, $23,24]$. However, the D-dimer test is not helpful 
Table 2 Learning points regarding tests used in the diagnosis of pulmonary embolism (PE)

\begin{tabular}{|c|c|}
\hline CXR & $\begin{array}{l}\text { Normal CXR } \\
\text { Plate atelectasis } \\
\text { Hampton hump (pleural-based opacity) } \\
\text { Small pleural effusion } \\
\text { Elevated hemidiaphragm } \\
\text { Fleischner's sign (prominent amputated pulmonary artery) } \\
\text { Westermark's sign (peripheral oligaemia) } \\
\text { The more abnormal the CXR, the less likely is PE } \\
\text { Normal CXR in a breathless hypoxic person in the absence of bronchospasm means that PE is likely }\end{array}$ \\
\hline ECG & $\begin{array}{l}\text { Sinus tachycardia } \\
\text { Nonspecific T-wave changes } \\
\text { P-pulmonale } \\
\text { RV strain } \\
\text { Right bundle branch block } \\
\text { S1, Q3, T3 (deep S-wave in lead I, Q-wave in lead II and T-wave inversion in lead III) } \\
\text { ECG is very useful at revealing alternative diagnoses (e.g. myocardial infarction) }\end{array}$ \\
\hline ABGs & $\begin{array}{l}\text { Hypoxaemia, hypocapnia and increased } P \mathrm{~A}-\mathrm{a}, \mathrm{O}_{2} \\
\text { Can be normal in } \mathrm{PE} \text {, especially in young people with good pulmonary reserve }\end{array}$ \\
\hline D-dimer & $\begin{array}{l}\text { D-dimer should always be considered with the clinical probability } \\
\text { Negative } D \text {-dimer is useful in excluding PE in the setting of low clinical probability and obviate the need for further } \\
\text { imaging. } \\
\text { D-dimer is not recommended to be used when the clinical probability of PE is high, as it is unlikely to influence the } \\
\text { decision for further imaging and would most likely be positive. }\end{array}$ \\
\hline CUS & Leg ultrasound study can be helpful as an adjunctive test to nondiagnostic imaging ( $V^{\prime} / Q^{\prime}$ or CTPA) in diagnosis of PE. \\
\hline$V^{\prime} / Q^{\prime}$ & $\begin{array}{l}\text { A high } V^{\prime} / Q^{\prime} \text { probably indicates that PE is very likely, especially when combined with a high clinical probability. } \\
\text { Normal or near-normal } V^{\prime} / Q^{\prime} \text { scan virtually excludes } P E \text {. } \\
\text { Nondiagnostic scans occur in most of the patients undergoing } V^{\prime} / Q^{\prime} \text { scanning, especially when there is } \\
\text { cardiopulmonary disease or abnormal CXR; these patients should be investigated further }\end{array}$ \\
\hline CTPA & $\begin{array}{l}\text { CTPA is easier to read than } V^{\prime} / Q^{\prime} \text { scans, even in the presence of cardiopulmonary disease or abnormal CXR; CTPA has } \\
\text { now replaced } V^{\prime} / Q^{\prime} \text { scanning as the screening diagnostic test for PE in many institutions } \\
\text { The diagnosis of PE using CTPA can be improved if CUS is used as an adjunctive test and clinical probability is taken } \\
\text { into account. } \\
\text { It is safe to withhold anticoagulant therapy after a negative CTPA and a negative CUS if the clinical probability is low. } \\
\text { It is also probably safe to withhold anticoagulant therapy after a negative CTPA and a negative CUS with intermediate } \\
\text { clinical probability, although this approach should be considered with caution. } \\
\text { The chance of missing PE with a negative CTPA and a negative CUS in patients with high clinical probability is } \\
\text { relatively high and further evaluation is warranted in these patients. }\end{array}$ \\
\hline Troponin-T and -I & $\begin{array}{l}\text { Can be raised in severe PE } \\
\text { Can not be used to rule out PE, but can be used in risk stratification of PE to identify low-risk patients with PE who } \\
\text { can be treated as outpatients }\end{array}$ \\
\hline BNP & $\begin{array}{l}\text { Elevated levels of BNP are associated with RV dysfunction in PE } \\
\text { It can be used in risk stratification of PE severity }\end{array}$ \\
\hline
\end{tabular}

in the context of a high clinical probability of $\mathrm{PE}$, as a low value would not exclude the diagnosis and subsequent investigations would be required. In this situation, the D-dimer test is best avoided. Recently, Douketis et al. [25] conducted a meta-analysis in 1,818 patients with unprovoked $P E$ in order to determine whether the of postanticoagulation timing of D-dimer testing, patient age and the cutoff point used to define a positive or negative result affect the ability of this test to distinguish risk for recurrent disease. Although theses authors found that the risk for recurrent VTE was higher in patients with a positive D-dimer result than in those with a negative result, this was not affected by the timing of postanticoagulation D-dimer testing, patient age or the assay cutoff point used. These results may suggest that D-dimer may be also used to determine the duration of anticoagulation and those who may need prolonged anticoagulation, though the risk of recurrent VTE has to be counterbalanced by the risk of major haemorrhage, especially in elderly [25].

\section{CUS of the lower limbs}

Better sensitivity and specificity have resulted in serial CUS of the lower limbs replacing contrast venography in the diagnosis of deep venous thrombosis (DVT) [6-7, 26-28]. Although most cases of PE are believed to arise from the proximal veins of the lower limbs, CUS is positive 


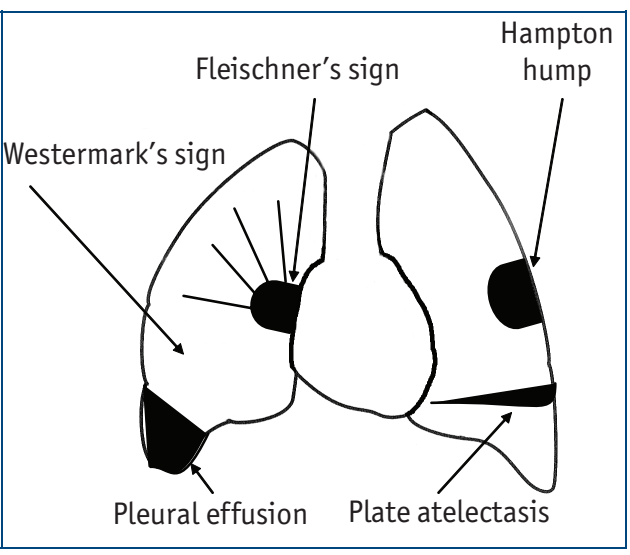

Figure 2

Abnormalities that can be detected in chest radiography in patients with pulmonary embolism. Hampton hump indicates pleural-based opacity (pulmonary infarction), Fleischner's sign indicates prominent amputated pulmonary artery and Westermark's sign indicates peripheral oligaemia.

in only onethird of patients with proven PE [29]. Possible explanations for negative CUS in patients with PE include other sources of emboli (e.g. upper $\operatorname{limb}$ ), intravascular nonoccluding thrombus, an already dislodged leg thrombus and falsenegative studies [30]. Despite its low sensitivity in the diagnosis of thrombosis in patients with $\mathrm{PE}, \mathrm{CUS}$ can still play a role in the diagnostic work-up of PE and can be used as an adjunctive test in case of nonconclusive isotope lung imaging or negative CTPA [31, 32].

\section{$V^{\prime} / Q^{\prime}$ isotope scan}

The Prospective Investigation of Pulmonary Embolism Diagnosis (PIOPED) study established that the likelihood of a PE diagnosis with a high $V^{\prime} / Q^{\prime}$ isotope scan probability is high, at $87 \%$ [33]. However, a normal or near-normal $V^{\prime} / Q^{\prime}$ scan has a high negative predictive value and virtually excludes PE [33]. Furthermore, the predictive value of $V^{\prime} / Q^{\prime}$ scanning can be further improved when considered in the context of clinical probability. For example, the likelihood of PE could be increased to from $87 \%$ to $96 \%$ if the high $V^{\prime} / Q^{\prime}$ probability is combined with a high clinical probability [33]. Conversely, the likelihood of PE could be decreased from 14\% to $4 \%$ if the low $V^{\prime} / Q^{\prime}$ probability is combined with a low clinical probability [33]. However, most patients (57\%) will have nondiagnostic $V^{\prime} / Q^{\prime}$ (intermediate, low or indeterminate probability), especially in those with abnormal CXR or cardiopulmonary disease, which necessitate further adjunctive tests.

\section{CTPA}

CTPA scanning has many advantages over $V^{\prime} / Q^{\prime}$ scanning as a screening diagnostic test for $\mathrm{PE}$, being quicker to perform, easier to read, less affected by the presence of cardiopulmonary disease or abnormal CXR, and being able to give an alternative diagnosis in many cases $[34,35]$. Furthermore, CTPA provides a quantitative assessment of $P E$, which correlates well with the severity of the clinical picture $[34,35]$. In addition, computerised tomographic venography of the iliac veins and the inferior vena cava can be performed simultaneously with the lung scan, and can have a high sensitivity (97\%) and specificity $(100 \%)$ with no additional contrast, although it involves a significant additional radiation dose to the reproductive organs, especially in a young person [36].

Studies have demonstrated variable sensitivities and specificities of CTPA. QANADLI et al. [37], using pulmonary angiography as a reference gold standard test $(n=157)$, found that the specificity and sensitivity of CTPA approached 94\% and 90\%, respectively. However, PeRRIER et al. [38] studied the diagnostic value of CTPA in 287 patients with suspected PE and a positive $\mathrm{D}$ dimer test, using a high-probability $V^{\prime} / Q^{\prime}$ scan, a positive CUS plus a clinical suspicion of $P E$, or positive pulmonary angiography as reference tests. Those authors reported lower CTPA specificity (91.2\%) and sensitivity (70\%). In particular, 35 patients with PE had negative CTPA, and the diagnosis of PE in these patients was established by other tests [38]. These findings suggest caution in the interpretation of CTPA; specifically, it should not be used alone to rule out PE. Subsequent investigators studied the safety of withholding anticoagulant therapy following negative findings on CTPA plus CUS. Musset et al. [32] investigated 1,041 patients with suspected PE in a large, prospective, multicentre study involving 14 French hospitals using singledetector CTPA, and demonstrated that PE was likely to be missed with negative testing on both CTPA and CUS in $1.8 \%$ of patients with low or intermediate clinical probability, compared with $5.3 \%$ of patients with high clinical probability. Similarly, PERRIER et al. [39], in a more recent study of 756 patients with suspected PE using multi-detector CTPA, found that there was a $1.7 \%$ likelihood of missing VTE among patients with positive D-dimer, and low or intermediate clinical probability when the CTPA and CUS were negative [39]. More recently, PIOPED II investigated the role of multidetector CTPA in the diagnosis of PE 
in 773 patients, using a composite reference standard to rule out $P E$, including $V^{\prime} / Q^{\prime}$ scan showing a high probability of $P E$, a positive digital subtraction angiogram or a positive CUS with a nondiagnostic $V^{\prime} / Q^{\prime}$ scan. Patients with negative results for PE were followed for 6 months. This study demonstrated that multidetector CT-PA had a sensitivity of $83 \%$ and specificity of $96 \%$. The same study demonstrated that the sensitivity of multidetector CTPA, when combined with venous-phase imaging, increased to $90 \%$, with $95 \%$ specificity [40]. The same study demonstrated that the predictive value of CTPA varied substantially when the clinical assessment was taken into account, with respective negative predictive values for exclusion of PE for high, intermediate and low clinical probability of 60,89 and $96 \%$ for CT-PA alone, and 82,92 , and 97\% for CTPA combined with venous phase imaging, respectively. Although PIOPED II showed the predictive value of either CTPA alone or combined with venous imaging to be high with a concordant clinical assessment, additional testing, including $V^{\prime} / Q^{\prime}$ scan, seemed to be necessary when clinical probability was inconsistent with the imaging results [40]. In addition, CTPA proved to be the preferred investigation in massive (hypotensive acute PE) and submassive (normotensive acute PE with right ventricular (RV) dysfunction) PE [41-42]. Early identification of these patients is of pivotal importance, as institution of thrombolytic therapy, surgical embolectomy or catheter-assisted embolectomy can be life-saving [43]. CTPA in this setting is especially useful, as it may show the location (central location) and size of the clot, as well as demonstrating features indicative of RV dysfunction. These features include right ventricle size larger than the size of left ventricle, bulging of the interventricular septum to the left and a large pulmonary artery diameter that may exceed the aortic diameter [34, 35]. Other investigations that can be helpful in this situation include echocardiogram and cardiac markers, such as troponin and B-type natriuretic peptuide (BNP). Additionally, CTPA is a very useful test in the diagnosis of chronic thromboembolic pulmonary hypertension, and in evaluating the suitability of such patients for surgery and pulmonary endarterectomy [44].

\section{Pulmonary angiography}

With the introduction of CTPA, the use of pulmonary angiography has become very limited. It is usually indicated when there is a high clinical suspicion of PE with nondiagnostic scan, and the diagnosis can not be established by less invasive tests [6-8]. Although pulmonary angiography is regarded as the gold standard diagnostic technique for $\mathrm{PE}$, it has not been tested against a reference test and established its position by default. In fact, studies demonstrated that recurrent thromboembolic events were described following normal angiographic results [4]. Adverse effects related to pulmonary angiography include death $(0.2-0.5 \%)$, severe cardiopulmonary compromise $(0.4 \%)$, renal failure requiring dialysis $(0.3 \%)$, elevation in the serum creatinine without the need for dialysis $(0.9 \%)$ and groin haematomas requiring transfusion $(0.2 \%)[6,8,33]$.

\section{Cardiac biomarkers}

\section{Troponin}

Troponin-T or -I can be raised in acute PE secondary to myocyte injury in the right ventricle, which correlates with the presence of RV dysfunction, cardiogenic shock and in-hospital mortality $[45,46]$. Raised troponin usually indicates massive or submassive $\mathrm{PE}$, although it can be elevated in patient with small PE $[42$, 45-47]. Unlike D-dimer, troponin cannot be used to rule out PE in the acute clinical setting, but can serve as a clue to the diagnosis of $\mathrm{PE}$, and can be used in risk stratification of PE to identify low-risk patients with PE who can be treated as outpatients, especially if combined with other tests. Recently, JIMÉNEZ and co-workers $[47,48]$ have found that the combination of positive troponin 1 with either DVT detected by CUS or RV dysfunction detected by transthoracic echocardiogram are associated with a PE-related mortality of $17.1 \%$ and $15.2 \%$, respectively.

\section{BNP}

BNP levels rise in response to stretch and/or increased ventricular pressure. Elevated levels of BNP are associated with RV dysfunction and RV failure, and it can be used to assess RV function in patients with PE. Indeed, a high BNP level has been shown to be associated with adverse outcome of PE $[47,49,50]$.

\section{Echocardiography}

Echocardiographic abnormalities observed in PE include RV dysfunction and tricuspid regurgitation [51-53]. Regional wall motion abnormalities that spare the RV apex (McConnell's sign) were found to be very suggestive of $\mathrm{PE}$, with $77 \%$ 
sensitivity and 95\% specificity [54]. Although transoesophageal echocardiography has been used successfully to detect PE in the right heart and main pulmonary artery [55], echocardiography has low specificity for PE and is not normally used as an initial screening test for this condition. However, echocardiography can be very useful in evaluating the severity of $\mathrm{PE}$, especially if thrombolytic therapy is contemplated $[47,48,56,57]$.

\section{Diagnostic approach}

All patients with suspected PE should be evaluated for clinical probability (fig. 3). If the clinical probability is low, D-dimer should be measured. Patients with a negative D-dimer test result, orlow pre-test clinical probability plus negative D-dimer are unlikely to have PE and

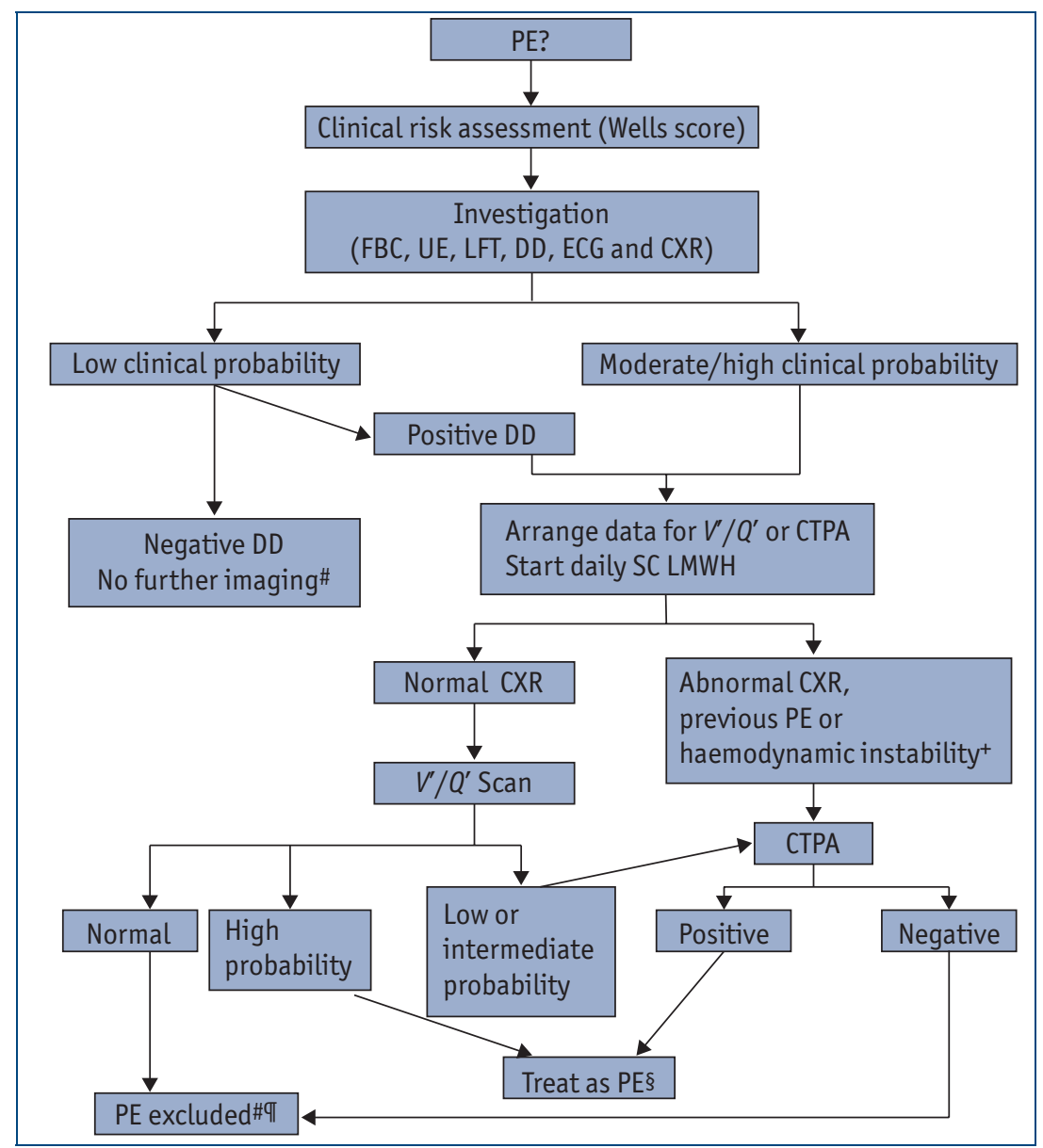

Figure 3

Diagnostic algorithm of pulmonary embolism (PE) at James Cook University Hospital (Middlesborough, UK). FBC: full blood count; UE: urea and electrolytes; LFT: liver function tests; DD: D-dimer; CXR: chest radiography; $V^{\prime} / Q^{\prime}$ : ventilation/perfusion scan; CTPA: computerised tomographic pulmonary angiography; SC LMWH: subcutaneous low molecular weight heparin. \#: consider alternative diagnosis; " suspicion high; ${ }^{+}$: consider echocardiogram (possible right ventricular strain); ${ }^{\S}$ : commence warfarin, international normalised ratio 2-2.5 for 6 months if first episode. need no further tests. However, patients with an intermediate or high clinical probability, or low clinical probability plus positive D-dimer test results should either have a $V^{\prime} / Q^{\prime}$ scan or CTPA screening test, depending on CXR findings. It is generally recommended that in order to reduce costs, patients with normal CXR have a $V^{\prime} / Q^{\prime}$ scan, while CTPA is reserved for patients with abnormal CXR or cardiopulmonary disease.

A normal $V^{\prime} / Q^{\prime}$ scan virtually excludes $P E$ without the need for further tests. In contrast, a high $V^{\prime} / Q^{\prime}$ probability scan is highly suggestive of PE especially when combined with high clinical probability. A nondiagnostic $V^{\prime} / Q^{\prime}$ scan (low or intermediate probability) should be supplemented by further testing, such as a lower limb study, CTPA or angiography, depending on the clinical probability. However, patients with low $V^{\prime} / Q^{\prime}$ scan probability plus low clinical probability are less likely to have $P E$, and probably no further testing is required.

A negative CTPA combined with a negative CUS virtually excludes $P E$, especially if the clinical probability is low. A negative CTPA combined with a negative CUS does not confidently exclude PE in patients with high clinical probability, and further testing may be needed.

It should be emphasised here that the diagnostic approach advocated here, as well as those mentioned elsewhere, may need to be adapted according the individual patient's presentation. Consequently, clinicians may be faced with difficult cases where they have to use their own discretion and clinical experience.

\section{Learning lessons from cases presented to Acute Assessment Unit, James Cook University Hospital (Middlesborough, UK)}

\section{Case 1}

A 27-yrold female admitted to Acute Assessment Unit via the Accident and Emergency (A\&E) department with right pleuritic chest pain, haemoptysis and breathlessness. She was taking the oral contraceptive pill and had a family history of PE (mother and sister). She was a social smoker. Upon examination, her pulse was 83 beats. $\mathrm{min}^{-1}$, temperature was $36^{\circ} \mathrm{C}$, oxygen saturation was $97 \%$ in room air and blood 


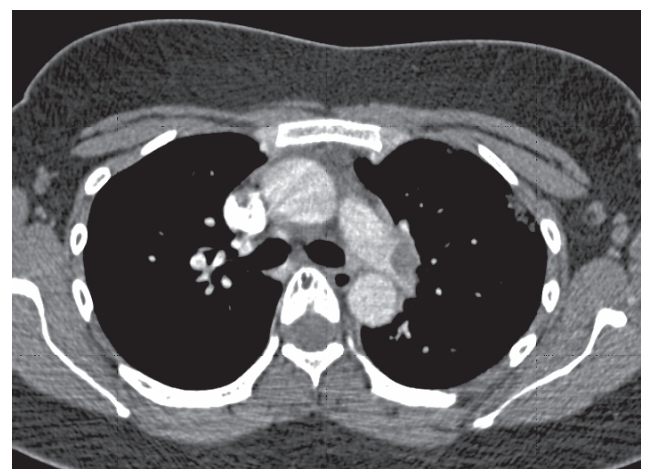

Figure 4

Computerized tomographic pulmonary angiography of case 1 , showing a filling defect in the left main pulmonary artery.

pressure $149 / 95 \mathrm{mmHg}$. The rest of clinical examination was unremarkable. Investigations showed normal $A B G$, full blood count $(F B C)$, urea and electrolytes. CXR was normal, while Ddimer was high. Clinical probability of PE was determined to be high. $V^{\prime} / Q^{\prime}$ scan was reported as low PE probability. She was discharged with a diagnosis of musculoskeletal pain. She was referred to the Chest Clinic, where she had repeat CXR, which was normal, and she was reassured and discharged.

She was re-admitted 1 month later with severe right and leftsided pleuritic chest pain (requiring morphine), and breathlessness. Clinical examination revealed left-sided pleural rub. CXR was again normal. CTPA revealed a filling defect in left main pulmonary artery, consistent with PE (fig. 4).

\section{Case 2}

A 38-year-old, known asthmatic female was admitted via A\&E with leftsided pleuritic chest pain, but no breathlessness or cough. She was taking no medications apart from inhalers. Clinical examination was normal apart from tenderness at the site of the pain. Investigations showed normal $\mathrm{FBC}$, urea and electrolytes, C-reactive protein $<4 \mathrm{mg} \cdot \mathrm{L}^{-1}$, normal CXR, and a high D-dimer level. She had a $V^{\prime} / Q^{\prime}$ scan, which showed multiple bilateral perfusion defects that were more marked on the left side, consistent with a high probability of PE with normal CXR appearance (fig. 5). CTPA was arranged and was completely normal. Patient was reassured and discharged.

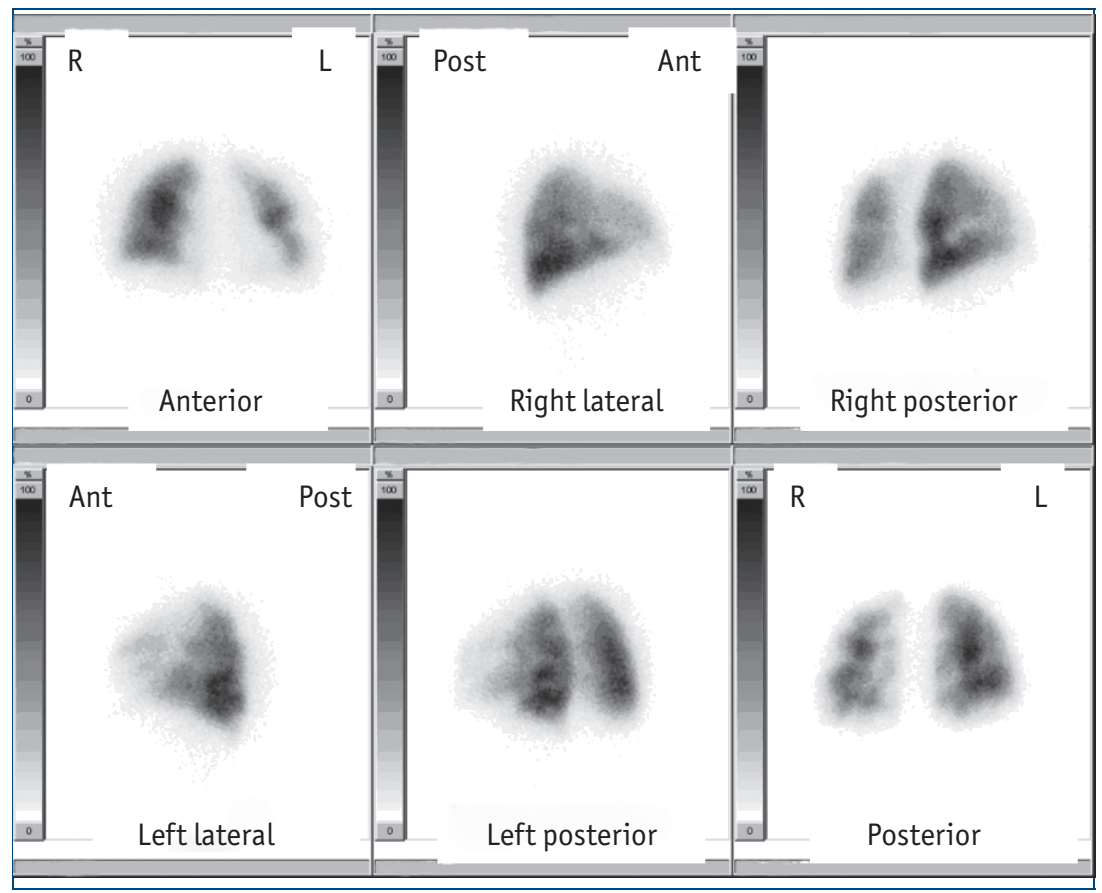

Figure 5

Ventilation/perfusion scan of case 2, showing multiple perfusion defects. L: left; R: right; Ant: anterior; Post: posterior.

\section{Discussion}

In case 1, there was a high clinical probability for $P E$ (presence of risk factors and no convincing alternative diagnosis). $\mathrm{ABG}$ can be normal in $\mathrm{PE}$, especially in young people with good pulmonary reserve, as in this case. Low $V^{\prime} / Q^{\prime}$ probability does not exclude PE in the presence high or intermediate clinical probability, and further imaging was required (CTPA).

In contrast, the clinical probability for PE was low in case 2, because there was no risk factor and the tenderness suggested muscloskeletal pain (presence of an alternative diagnosis). However, the presence of high D-dimer levels justified further imaging $\left(V^{\prime} / Q^{\prime}\right.$ scan). The presence of multiple perfusion defects in the $V^{\prime} / Q^{\prime}$ scan is a common occurrence in asthma and chronic obstructive pulmonary disease, as bronchoconstriction causes area of hypoxia and $\mathrm{CO}_{2}$ retention, leading to vasoconstriction as a compensatory mechanism. Consequently, CTPA, rather than $V^{\prime} / Q^{\prime}$ scan, would have been more appropriate investigation in this case.

\section{References}

1. Dalen JE, Alpert JS. Natural history of pulmonary embolism. Prog Cardiovasc Dis 1975; 17: 259-270.

2. Hyers TH. Venous thromboembolism. Am J Respir Crit Care Med 1999; 159: 1-14.

3. Goldhaber SZ. Pulmonary embolism. Lancet 2004; 363: 1295-1305. 
4. van Beek EJ, Brouwerst EM, Song B, et al. Clinical validity of a normal pulmonary angiogram in patients with suspected pulmonary embolism - a critical review. Clin Radiol 2001; 56: 838-842.

5. Hyers TM. Diagnosis of pulmonary embolism. Thorax 1995; 50: 930-932.

6. Tapson VF, Carroll BA, Davidson BL, et al. The diagnostic approach to acute venous thromboembolism. Clinical practice guideline. American Thoracic Society. Am J Respir Crit Care Med 1999; 160: 1043-1066.

7. Suspected acute pulmonary embolism. a practical approach. British Thoracic Society, Standards of Care Committee. Thorax 1997; 52: Suppl. 4, S1-S24.

8. British Thoracic Society Standards of Care Committee Pulmonary Embolism Guideline Development Group. British Thoracic Society guidelines for the management of suspected acute pulmonary embolism. Thorax 2003; 58: 470484.

9. Wells PS, Ginsberg JS, Anderson DR, et al. Use of a clinical model for safe management of patients with suspected pulmonary embolism. Ann Intern Med 1998; 129: 997-1005.

10. Wells PS, Anderson DR, Rodger M, et al. Excluding pulmonary embolism at the bedside without diagnostic imaging: management of patients with suspected pulmonary embolism presenting to the emergency department by using a simple clinical model and D-dimer. Ann Intern Med 2001; 135: 98-107.

11. Wells PS, Anderson DR, Rodger M, et al. Derivation of a simple clinical model to categorize patient probability of pulmonary embolism: increasing the models utility with the SimpliRED D-dimer. Thromb Haemost 2000; 83: 416420.

12. Davies CWH, Bell D, Wimperis J, et al. Validation of pretest probability (PTP) scored to predict pulmonary embolism in routine practice. Thorax 2003; 58: Suppl. 3, S82-S83.

13. Whitaker AN, Elms MJ, Massci PP, et al. Measurement of cross-linked fibrin derivatives in plasma: an immunoassay using monoclonal antibodies. J Clin Pathol 1984; 37: 882-887.

14. Hirsh J, Lee AYY. How we diagnose and treat deep venous thrombosis. Blood 2002; 99: 3102-3110.

15. Stein PD, Terrin ML, Hales CA, et al. Clinical, laboratory, roentgenographic, and electrocardiographic findings in patients with acute pulmonary embolism and no pre-existing cardiac or pulmonary disease. Chest 1991; 100: 598603.

16. Szucs MM, Brooks HL, Grossman W, et al. Diagnostic sensitivity of laboratory findings in acute pulmonary embolism. Ann Intern Med 1971; 74: 161-166.

17. Moser KM. Diagnosing pulmonary embolism. BMJ 1994; 309: 1525-1526.

18. Ginsberg JS, Brill-Edwards PA, Demers C, et al. D-dimer in patients with clinically suspected pulmonary embolism. Chest 1993; 104: 1679-1684.

19. de Moerloose P, Minazio P, Reber G, et al. D-dimer determination to exclude pulmonary embolism: a two step approach using latex assay as a screening tool. Thromb Haemost 1994; 72: 89-91.

20. Farrell S, Hayes T, Shaw M. A negative SimpliRED D-dimer assay result does not exclude the diagnosis of deep vein thrombosis or pulmonary embolus in emergency department patients. Ann Emerg Med 2000; 35: 121-125.

21. Ginsberg JS, Wells PS, Kearon C, et al. Sensitivity and specificity of a rapid whole-blood assay for D-dimer in the diagnosis of pulmonary embolism. Ann Intern Med 1998; 129: 1006-1011.

22. Bates SM, Grand Maison A, Johnston M, et al. A latex D-dimer reliably excludes venous thromboembolism. Arch Intern Med 2001; 161: 447-453.

23. Perrier A, Desmarais S, Miron MJ, et al. Non-invasive investigation of VTE in outpatients. Lancet 1999; 353: 190195.

24. Gandra E, Wells PS. Diagnosis: use of clinical probability algorithms. Clin Chest Med 2010; 31: 629-639.

25. Douketis JD, Gu CS, Schulman S, et al. The risk for fatal pulmonary embolism after discontinuing anticoagulant therapy for venous thromboembolism. Ann Intern Med 2007; 147: 766-774.

26. Heijboer $\mathrm{H}$, Buller HR, Lensing AWA, et al. A comparison of real-time compression ultrasonography with impedance plethysmography for the diagnosis of deep-vein thrombosis in symptomatic outpatients. N Engl J Med 1993; 329: $1365-1369$.

27. Birdwell BG, Raskob TL, Whitsett TL, et al. The clinical validity of normal compression ultrasonography in outpatients suspected of deep venous thrombosis. Ann Intern Med 1998; 128: 1-7.

28. Cogo A, Lensing AW, Koopman MM, et al. Compression ultrasonography for diagnostic management of patients with clinically suspected deep vein thrombosis: prospective cohort study. BMJ 1998; 316: 17-20.

29. Turkstra F, Kuijer PM, van Beck EJ, et al. Diagnostic utility of ultrasonography of leg veins in patients suspected of having pulmonary embolism. Ann Intern Med 1997; 126: 775-781.

30. Davidson BL. Controversies in pulmonary embolism and deep venous thrombosis. Am Fam Physician 1999; 60: 1969-1980.

31. Meyerovitz MF, Manning F, Polak JF, et al. Frequency of pulmonary embolism in patients with low probability lung scan and negative lower extremity venous ultrasound. Chest 1999; 115: 980-982.

32. Musset D, Parent F, Meyer G, et al. Diagnostic strategy for patients with suspected pulmonary embolism: a prospective multicentre outcome study. Lancet 2002; 360: 1914-1920.

33. Value of the ventilation/perfusion scan in acute pulmonary embolism. Results of the prospective investigation of pulmonary embolism diagnosis (PIOPED). The PIOPED Investigators. JAMA 1990; 263: 2753-2759.

34. Cuteo SM, Cavanaugh SH, Benenson RS, et al. Computed tomography scan versus ventilation-perfusion lung scan in the detection of pulmonary embolism. J Emerg Med 2001; 21: 155-164.

35. Bankier AA, Janata K, Fleischmann D, et al. Severity assessment of acute pulmonary with spiral CT: evaluation of two modified angiographic scores and comparison with clinical data. J Thoracic Imaging 1997; 12: 150-158.

36. Srivastava SD, Eagleton MJ, Greenfield LJ. Diagnosis of pulmonary embolism with various imaging modalities. Semin Vasc Surg 2004; 17: 173-180.

37. Qanadli SD, Hajjam ME, Mesurolle B, et al. Pulmonary embolism detection: prospective evaluation of dual-section helical CT versus selective pulmonary arteriography in 157 patients. Radiology 2000; 217: 447-455.

38. Perrier A, Howarth N, Didier D, et al. Performance of helical computed tomography in unselected outpatients with suspected pulmonary embolism. Ann Int Med 2001; 135: 88-97.

39. Perrier A, Roy PM, Sanchez 0 , et al. Multidetector-row computed tomography in suspected pulmonary embolism. $N$ Engl J Med 2005; 352: 1760-1768.

40. Stein PD, Fowler SE, Goodman LR, et al. Multidetector computed tomography for acute pulmonary embolism. N Engl J Med 2006; 354: 2317-2327. 
41. Kucher N, Rossi E, De Rosa M, et al. Massive pulmonary embolism. Circulation 2006; 113: 577-582.

42. Piazza G, Goldhaber SZ. Fibrinolysis for acute pulmonary embolism. Vasc Med 2010; 15: 419-428.

43. Ocak I, Fuhrman C. CT angiography findings of the left atrium and right ventricle in patients with massive pulmonary embolism. Am J Roentgenol 2008; 191: 1072-1076.

44. Pepke-Zaba J. Diagnostic testing to guide the management of chronic thromboembolic pulmonary hypertension: state of the art. Eur Respir Rev 2010; 19: 55-58.

45. Giannitsis E, Muller-Bardorff M, Kurowski V, et al. Independent prognostic value of cardiac troponin $T$ in patients with confirmed pulmonary embolism. Circulation 2000; 102: 211-217.

46. Meyer T, Binder L, Hruska N, et al. Cardiac troponin I elevation in acute pulmonary embolism is associated with right ventricular dysfunction. J Am Coll Cardiol 2000; 36: 1632-1636.

47. Jiménez D, Aujesky D, Yusen RD. Risk stratification of normotensive patients with acute symptomatic pulmonary embolism. Br J Haematol 2010; 151: 415-424.

48. Jiménez D, Aujesky D, Moores L, et al. Combination of prognostic tools for identification of high risk normotensive patients with acute symptomatic pulmonary embolism. Thorax 2011; 66: 75-81.

49. Kucher N, Goldhaler SZ. Risk stratification of acute pulmonary embolism. Semin Thrombo Hemost 2006; 32: 838847.

50. Binder $\mathrm{L}$, Pieske B, Olschewski $\mathrm{M}$, et al. $\mathrm{N}$-terminal pro-brain natriuretic peptide or troponin testing followed by echocardiography for risk stratification of acute pulmonary embolism. Circulation 2005; 13: 1520-1521.

51. Gibson NS, Sohne M, Buller HR. Prognostic value of echocardiography and spiral computed tomography in patients with pulmonary embolism. Curr Opin Pulm Med 2005; 11: 380.

52. Kucher N, Rossi E, De Rosa M, et al. Prognostic role of echocardiography among patients with acute pulmonary embolism and a systolic arterial pressure of $90 \mathrm{~mm} \mathrm{Hg}$ or higher. Arch Intern Med 2005; 165: 1777.

53. Come PC. Echocardiographic evaluation of pulmonary embolism and its response to therapeutic interventions. Chest 1992; 101: 151S.

54. Grifoni S, Olivotto I, Cecchini P, et al. Short-term clinical outcome of patients with acute pulmonary embolism, normal blood pressure, and echocardiographic right ventricular dysfunction. Circulation 2000; 101: 2817.

55. McConnell MV, Solomon SD, Rayan ME, et al. Regional right ventricular dysfunction detected by echocardiography in acute pulmonary embolism. Am J Cardiol 1996; 78: 469.

56. Pruszczyk P, Torbicki A, Pacho R, et al. Noninvasive diagnosis of suspected severe pulmonary embolism: transesophageal echocardiography versus spiral CT. Chest 1997; 112: 722.

57. Goldhaber SZ, Haire WD, Feldstein ML, et al. Alteplase versus heparin in acute pulmonary embolism: randomized trial assessing right ventricular function and pulmonary perfusion. Lancet 1993; 341: 507. 